\title{
Narrativa de las protagonistas trans en la teleserie Veneno
}

\author{
Trans protagonisten narratiba Veneno telesailean \\ Narrative of the trans protagonists in the TV series Veneno
}

\author{
Sergio Cobo-Durán^, Sofia Otero-Escudero \\ Universidad de Sevilla
}

RESUMEN: En este texto se estudia la construcción de las protagonistas trans en la teleserie Veneno (2020). Primero, se realiza un análisis semiótico de los personajes y su entorno centrado en tres niveles (persona, rol y actante). Posteriormente y tras una revisión bibliográfica se esboza una ficha de análisis que es aplicada en todos los episodios. Finalmente, se observan las características principales a través de un estudio apoyado en el análisis textual filmico de escenas clave. Así, se concluye cómo la serie analizada, a excepción de ciertos aspectos positivos en el personaje de Valeria, perpetúa una construcción canónica del colectivo trans.

PALABRAS CLAVE: serie de televisión; trans; personajes; narrativa; Veneno.

ABSTRACT: The present work scrutinizes the construction of the trans protagonists in the TV series Veneno (2020). First, a semiotic analysis of the characters and their environment is carried out, centered on three levels (person, role, and actant). Second, an analysis sheet is outlined to apply it in each of the episodes. Finally, the main characteristics are observed through a study of some key scenes. This way, it is concluded how the TV series analyzed perpetuates a canonical construction of the trans collective except for certain positive aspects depicted through Valeria's character.

KEYWORDS: TV series; Trans; Characters; Narrative; Veneno.

\footnotetext{
* Correspondencia a / Corresponding author: Sergio Cobo-Durán. Facultad de Comunicación. Universidad de Sevilla (41092 Sevilla, ES, España) - cobosergio@us.es - https://orcid.org/0000-0003-0374-6601

Cómo citar / How to cite: Cobo-Durán, Sergio; Otero-Escudero, Sofía (2021). "Narrativa de las protagonistas trans en la teleserie Veneno», Zer, 26(51), 79-97. (https://doi.org/10.1387/zer.23007).

Recibido: 29 julio, 2021; aceptado: 2 noviembre, 2021.

ISSN 1137-1102 - eISSN 1989-631X / (c) 2021 UPV/EHU

(c) (i) $(\$) \Theta$ Esta obra está bajo una Licencia

Cr ${ }_{\mathrm{NC}}$ Creative Commons Atribución-NoComercial-SinDerivadas 4.0 Internacional
} 


\section{Introducción}

Desde el inicio del siglo xxI, la representación de las personas trans en los medios de masas y la cultura popular ha aumentado la visibilidad del colectivo en la sociedad. No obstante, este aumento de la inclusión de personajes trans en los medios de comunicación no ha conllevado una construcción positiva, sino que se han perpetuado los estereotipos negativos. Las teorías y estudios que abordan las prácticas de representación de los colectivos, como plantea la presente investigación, son cruciales cuando se busca reflexionar sobre la construcción de personajes trans y las identidades que se presentan a la audiencia, haciendo hincapié en la posibilidad de representar al colectivo a través de narrativas alejadas de los estereotipos comunes. De este modo, se busca transformar el orden social, a través del desmantelamiento del sistema subyacente que permite que unas personas existan y otras sean condenadas a la discriminación o incluso a la invisibilidad (hooks, 1984; Stryker, 2006, 2017).

Gracias a diversos movimientos activistas y las circunstancias históricas detonantes, junto al impulso de teorías como el feminismo postcolonial o la teoría queer, en 1990 los estudios trans acceden a la agenda cultural y teórica para darle visibilidad a aquellos colectivos que llevaban décadas luchando por sus derechos desde el activismo (Stryker, 2006, 2017; Steinbock, 2016). Con la emergencia e impulso de estas teorías, surge el debate sobre el valor de la interseccionalidad y se pone el foco en el sistema heteronormativo que establece una serie de normas respecto al género, y provocan situaciones de violencia hacia las personas que no encajan en los patrones de género y sexualidad donde la heterosexualidad se establece como norma (López, 2017). Movimientos como el pensamiento queer, el transfeminismo o el feminismo postcolonial, cuestionan y reflexionan sobre las políticas de identidades y la libertad de cada individuo para elegir y cultivar su identidad fuera de estas exigencias establecidas. Autoras destacadas como Monique Wittig o Judith Butler definen la heterosexualidad obligatoria como mecanismo de poder y buscan crear espacios de resistencia ante esa orientación sexual impuesta desde diferentes ámbitos (Butler, 2006; Córdoba et al., 2005; López, 2017).

Así, mientras el concepto de género se va ampliando y evolucionando, aparece un nuevo cuestionamiento en ciertos sectores del feminismo sobre qué es ser mujer y qué es ser feminista, con grupos que comienzan a excluir a las identidades trans de sus espacios de lucha y reivindicación (Crenshaw, 1991; Green, 2006; Halberstam, 2016; hooks, 1984; M.Rodemeyer, 2018; Stryker, 2017).

Ante esta división interna y discriminación hacia el colectivo, surge uno de los ensayos fundacionales de los estudios trans, redactado por la artista y teórica Sandy Stone en 1992, The Empire Strikes Back: A Posttransexual Manifesto. El manifiesto propone que no se constituya a la persona trans como un problemático 'tercer género', sino como un género que busca alterar y producir sexualidades estructuradas 
(Enke, 2012; Stone, 1992; Stryker y Bettcher, 2016; Stryker, 2017). En ese mismo año, el término transgénero (transgender) obtiene su definición real tras la publicación de Leslie Feinberg, Transgender Liberation: A Movement Whose Time has Come. Posteriormente, la activista Virginia Prince, utiliza por primera ver el término para hacer referencia a las personas que se identificaban con una identidad de género dentro de un rango más amplio, sin ser travesti ni tampoco transexual. En definitiva, se empieza a utilizar como término paraguas que abarca cualquier tipo de expresión e identidad de género que se aleja de lo normativo y se deja de hacer alusión a las diferencias por modificaciones físicas — referido con el término transexual- ofreciendo la posibilidad de representar una mayor diversidad de corporalidades (Bernini, 2018; Halberstam, 2005; Stryker, 2006, 2017). Es crucial tener en cuenta la importancia del contexto respecto a la terminología, matizando que, aunque en Estados Unidos el término transgénero se haya usado como aglutinador desde los años 90 , en español es transexual y posteriormente trans $-\mathrm{O}$ trans ${ }^{\star}$ con asterisco-, el que se utiliza para hacer referencia al colectivo desde una perspectiva más amplia (Galofre y Missé, 2015; Stryker, 2017). Con estos términos más amplios, se abarca a cualquier persona cuya identidad transgreda la heteronormatividad binaria, alejándose de las connotaciones biológicas que la palabra transgénero y transexual conllevan. Además, el recurso del asterisco convierte el vocablo trans en un prefijo al cual se le puede añadir cualquier sustantivo, haciéndolo así un término más diverso y abierto a un espectro mucho más amplio de opciones identitarias (Platero, 2017; Stryker, 2017).

Más allá de los logros desde el activismo y la visibilidad del colectivo a través de la evolución del lenguaje, los estudios trans surgen de la necesidad de tratar a las personas trans como sujetos protagonistas desde un enfoque interdisciplinar, también dentro de la academia y en consecuencia en la sociedad (Horak, 2018; Steinbock, 2016). El sistema heteronormativo en la sociedad establece a través de las prácticas sociales y culturales una serie de normas que, entre otras cosas, jerarquizan los colectivos emplazando a ciertas personas en situaciones de privilegio, mientras otras quedan marginadas evitando que puedan enunciar cualquier tipo de discurso. Es por ello que, a pesar de su carácter incipiente y continua actualización, estos estudios se plantean como una nueva disciplina para interpretar y analizar distintos fenómenos desde una posición interna, desde experiencias únicas. Uno de los principales objetivos que se plantean es el de desviar la mirada del fenómeno trans como un simple objeto de estudio o área de interés, hacia su consideración como una vía para analizar y desmantelar el sistema y sus prácticas culturales de representación, creando nuevas narrativas (Adair et al., 2020; Steinbock, 2016; Stryker, 2006, 2017).

\subsection{LA INCLUSIÓN DE PERSONAJES TRANS EN FICCIÓN TELEVISIVA}

La representación en discursos de ficción de colectivos con poca visibilidad ha sido uno de los principales estandartes de algunas pioneras investigadores feministas. 
Las luchas feministas entendieron pronto que la forma en que eran representadas en las ficciones era importante para reivindicar otros tipos de feminidad. No obstante, entre las propias divisiones internas del feminismo, el movimiento trans ha quedado fuera de algunos espacios de representación, alentando en la actualidad un acalorado debate acerca de su inclusión o no en dicho movimiento. Esta escisión interna afecta también a los discursos ficcionales en cuanto a su representación. Hay que tener en especial consideración que «las identidades no surgen naturalmente de los cuerpos, sino que más bien se imponen en ellos y se naturalizan mediante políticas, retóricas e imágenes» (García, 2018, p. 89).

El presente texto nace de una cuestión esencial: ¿de qué forma son representados los personajes trans? Así pues, la aparición de textos académicos en los últimos años analiza de diferentes formas esta incipiente representación (Fellner, 2017; K.Gillig et al., 2018; Koch-Rein et al., 2020; Sandercock, 2015; Sears, 2008). «For the longest time trans representations were closely tied to narrow and problematic depictions of gender non-conforming people as either dangerous psychopaths and sexual predators $»^{1}$ (Koch-Rein et al., 2020, p. 2). En estos textos se concluye que en la mayoría de las obras audiovisuales el colectivo trans es representado a través de estereotipos negativos, estando asociado además con la transición o el cambio. En este sentido, el término transición se relaciona con un proceso continuado que está dirigido hacia una meta que implica el cambio de físico, normalmente en relación con operaciones genitales, adquiriendo así los atributos de género que el sistema binario y heteronormativo establecen como válidos para ser reconocibles (Carter, 2014; Garner, 2014). Por este motivo, autores como Platero (2014) o la autora Torras (2017) recurren al término tránsito en el ámbito académico para eliminar la connotación de cambio y hacer referencia al momento en el que la persona busca ser reconocida por la sociedad.

No obstante, es relevante destacar que la presencia de personas trans ha aumentado de forma considerable en las teleseries. Casos reconocidos como Orange is the New Black (Netflix, 2013-2019), Glee (FOX, 2009-2015), Sense8 (Netflix, 20152018) o Transparent (Amazon Studios, 2014-2017) han ayudado a esta representación. Si para algunas académicas la representación que realiza Glee a través de la comedia ha sido positiva, otras autoras más críticas han señalado justo lo contrario (Dubrofsky, 2013; Kociemba, 2010). Y es que la comedia alimenta el debate acerca de la idoneidad del formato para la visibilización, como este caso.

\footnotetext{
1 «A lo largo del tiempo, las representaciones trans estuvieron estrechamente unidas a representaciones problemáticas sobre las personas que no están conformes con su género, siendo representadas como personas psicópatas peligrosas o depredadoras sexuales» (Koch-Rein et al., 2020, p.2). Traducción propia.
} 


\section{Metodología y corpus}

Este estudio se centra en la serie de televisión Veneno (2020) dirigida por Javier Calvo y Javier Ambrossi, producida por ATRESplayer PREMIUM y distribuida en Estados Unidos por HBO MAX. La producción consiste en una serie biográfica centrada en la vida del personaje televisivo Cristina Ortíz Rodríguez, 'Veneno' y que aborda la creación, auge y decadencia de uno de los iconos televisivos pop de final de los noventa. La serie ganó el premio GLADD a la mejor serie en español, el Iris de la crítica 2020 y fue nominada para el galardón Queer Palm del Festival de Cannes y en los premios MIPCOM Diversity TV Excellence Awards. Asimismo, ganó el premio Ondas a la mejor interpretación femenina para tres actrices trans, Daniela Santiago, Jedet Sánchez e Isabel Torres. Hay que destacar en este sentido lo acertado de contar con actrices pertenecientes al colectivo para interpretar papeles trans, algo que parece obvio pero que no resulta habitual en las representaciones. Tal y como afirman las teóricas Keegan, Horak y Steinbock (2018), aunque hay un mayor interés y un aumento de representaciones de personajes trans, la mayoría son interpretados por actores y actrices cisgénero ${ }^{2}$, incluyendo así a los personajes en las narrativas ficcionales, pero a través de corporalidades que no son reales, además de no incluirles en el mercado laboral, lo cual no fomenta la visibilidad y mejora de condiciones reales del colectivo trans (Keegan, Horak y Steinbock, 2018). La elección de esta serie de televisión está motivada por la inexistencia, hasta la actualidad, de investigaciones previas que aborden el análisis de forma académica de la serie de televisión Veneno, no obstante, el interés que despierta sí que motiva la aparición de numerosos trabajos final de grado que se acercan a la serie (Navarro-Navarro, 2021 o Madrigal-López, 2021).

La hipótesis de partida de la presente investigación parte de trabajos académicos que abordan tanto la representación de personajes en ficción (Cobo-Durán, 2011; Zurián 2018), como la inclusión de personajes trans en la misma (Fellner, 2017; K.Gillig et al., 2018; Sandercock, 2015; Sears, 2008; Koch-Rein et al., 2020). Se plantea que la representación de personajes trans en la serie de ficción contemporánea Veneno responde a una construcción basada en estereotipos y lugares comunes, lo que convierte su inclusión en un elemento al servicio del espectáculo.

De esta hipótesis de partida se desgranan dos de los principales objetivos del presente trabajo:

1. Determinar los elementos identitarios que construyen a nivel narrativo a los personajes trans protagonistas en la serie de televisión Veneno.

\footnotetext{
${ }^{2}$ El término cis se utiliza como prefijo, el cual se añade al sustantivo género para «designar a personas cuya identidad de género asumida coincide con aquella que se les ha asignado al nacer, en función de criterios normativos de correspondencia entre características del denominado sexo biológico y la identidad de género» (Martínez-Guzmán, 2007, p. 82).
} 
2. Detectar si el empleo de algunos recursos audiovisuales contribuyen a la posible perpetuación de estereotipos.

Para alcanzar estos objetivos, la presente investigación se propone un análisis semiótico del personaje y su entorno centrado en tres niveles. En primer término, a nivel del relato se analizará al personaje como persona; en segundo lugar, se estudiará el nivel de la historia centrada en el personaje como rol, mientras que en tercer y último lugar se analizará al personaje desde el nivel de la fábula, es decir, el personaje como actante (Casetti y di Chio, 1997; Greimas 1971; Propp, 1998). A nivel metodológico se trabaja con el estudio de caso de la serie de televisión Veneno. Para ello, y según Collazos (2009), el estudio de caso se trata de un «tratamiento holístico», donde se encuentran "distintas miradas desde las diferentes disciplinas y ciencias sociales; en él confluyen distintos métodos cualitativos y cuantitativos» (Collazos, 2009, p.188). Con el propósito de centrar el objeto de estudio se han seleccionado a las dos protagonistas, 'Veneno' y Valeria. Al tratarse de un recorrido biográfico por la vida de Cristina Ortíz se incluyen diferentes edades y momentos vitales, por tanto, se ha optado - muestra por conveniencia - por seleccionar la etapa de su vida que supone la mayor parte del relato seriado, su edad adulta (20-30 años). Del mismo modo, con respecto a Valeria, el análisis se centra en su edad adulta.

En cuanto a las características a nivel del relato se analizarán las características iconográficas (tabla 1): edad, rasgos indiciales (apariencia física), rasgos artifactuales (vestimenta, ademanes, forma de hablar), transformaciones; características psicológicas: comportamiento, relación, pensamiento, estados anímicos, emociones, valores, sentimientos, evolución; y a nivel sociológico: clase social, nivel cultural, nivel económico, amistades/familia y orientación sexual. Respecto a la representación como rol y actante (tabla 1) se han seleccionado escenas clave de la serie donde se estudiarán algunos elementos que inciden en la trama. 
TABLA 1

Ficha de análisis 1

\begin{tabular}{|c|c|c|c|}
\hline \multicolumn{4}{|c|}{ Nivel de relato. Personaje como persona } \\
\hline \multirow{4}{*}{ Iconografia } & \multicolumn{2}{|l|}{ Edad } & \\
\hline & \multicolumn{2}{|c|}{ Rasgos indiciales: apariencia física } & \\
\hline & \multicolumn{2}{|c|}{ Rasgos artifactuales: Vestimenta, ademanes, forma de hablar } & \\
\hline & \multicolumn{2}{|c|}{ Transformación } & \\
\hline \multirow{5}{*}{ Psicología } & \multicolumn{2}{|l|}{ Comportamiento } & \\
\hline & \multicolumn{2}{|l|}{ Relación } & \\
\hline & \multicolumn{2}{|c|}{ Estados anímicos, emociones, sentimientos } & \\
\hline & \multicolumn{2}{|l|}{ Valores } & \\
\hline & \multicolumn{3}{|l|}{ Evolución } \\
\hline \multirow{5}{*}{ Sociología } & \multicolumn{3}{|l|}{ Clase social } \\
\hline & \multicolumn{3}{|l|}{ Nivel cultural } \\
\hline & \multicolumn{3}{|l|}{ Nivel económico } \\
\hline & \multicolumn{3}{|l|}{ Amistades/familia } \\
\hline & \multicolumn{3}{|l|}{ Orientación sexual } \\
\hline \multicolumn{4}{|c|}{ Nivel de la historia. Personaje como rol } \\
\hline \multicolumn{4}{|l|}{ Rol } \\
\hline \multicolumn{4}{|c|}{ Motivaciones y acciones } \\
\hline \multicolumn{4}{|c|}{ Nivel de la fábula. Personaje como actante } \\
\hline \multicolumn{4}{|l|}{ Sujeto } \\
\hline \multicolumn{4}{|l|}{ Objeto } \\
\hline \multicolumn{4}{|l|}{ Destinador } \\
\hline \multicolumn{4}{|l|}{ Destinatario } \\
\hline \multicolumn{4}{|l|}{ Ayudante } \\
\hline Oponente & & & \\
\hline
\end{tabular}

Fuente: elaboración propia.

En un primer visionado de la serie se realiza una doble ficha de análisis que se aplicará en cada uno de los episodios. Esta rejilla (tabla 2) incorpora y analiza elementos como la atracción hacia otros personajes y su orientación sexual, centrándose especialmente en la violencia (fisica, psicológica o estructural sistémica) tanto desde 
el perfil de víctima como el de provocadora de esta. Igualmente, se analiza la representación de las relaciones familiares, amistosas, las relaciones laborales y su relación con el entorno social.

TABLA 2

Ficha de análisis 2

\begin{tabular}{|c|c|c|c|c|}
\hline Atracción por otros personajes & Capítulos & Secuencias & $\begin{array}{c}\text { Recursos } \\
\text { audiovisuales }\end{array}$ & Descripción \\
\hline \multicolumn{5}{|l|}{ Heterosexual } \\
\hline \multicolumn{5}{|l|}{ Homosexual } \\
\hline Víctimas de violencia & Capítulos & Secuencias & $\begin{array}{c}\text { Recursos } \\
\text { audiovisuales }\end{array}$ & Descripción \\
\hline \multicolumn{5}{|l|}{ Física } \\
\hline \multicolumn{5}{|l|}{ Psicológica } \\
\hline \multicolumn{5}{|l|}{ Estructural-sistémica } \\
\hline Provocadoras de violencia & Capítulos & Secuencias & $\begin{array}{c}\text { Recursos } \\
\text { audiovisuales }\end{array}$ & Descripción \\
\hline \multicolumn{5}{|l|}{ Física } \\
\hline \multicolumn{5}{|l|}{ Psicológica } \\
\hline \multicolumn{5}{|l|}{ Estructural-sistémica } \\
\hline Relaciones familiares & Capítulos & Secuencias & $\begin{array}{c}\text { Recursos } \\
\text { audiovisuales }\end{array}$ & Descripción \\
\hline \multicolumn{5}{|l|}{ Positivas } \\
\hline \multicolumn{5}{|l|}{ Negativas } \\
\hline \multicolumn{5}{|l|}{ No existe } \\
\hline Amistades & Capítulos & Secuencias & $\begin{array}{c}\text { Recursos } \\
\text { audiovisuales }\end{array}$ & Descripción \\
\hline \multicolumn{5}{|l|}{ No tiene } \\
\hline \multicolumn{5}{|l|}{ Solo con otros personajes trans } \\
\hline \multicolumn{5}{|l|}{ Con personajes cisgénero } \\
\hline Parejas & Capítulos & Secuencias & $\begin{array}{c}\text { Recursos } \\
\text { audiovisuales }\end{array}$ & Descripción \\
\hline \multicolumn{5}{|l|}{ No tiene } \\
\hline Sí tiene (número y ocasional o recurrente) & & & & \\
\hline
\end{tabular}

Fuente: elaboración propia. 
Para Halberstam (2005) existe una diferencia elemental entre la predominancia de películas con personajes trans pero que evitan la 'mirada transgénero'. Una vez aplicadas estas rejillas de análisis se ha considerado relevante detenerse en algunas cuestiones formales que afectan a la composición y realización de la producción. Para ello se atenderá también a autores que trabajan con la focalización y el punto de vista en la construcción del relato (Branigan, 1984; Cuevas, 2001; Gennete, 1998). En este sentido, se propone un análisis en profundidad de dos tipos de escenas imprescindibles en el arco narrativo de los personajes (Seid, 2014): escena de presentación y escena de sexo. El patrón de análisis parte de la idea de que una gramática cinematográfica basada en una perspectiva cisgénero ofrece a la audiencia imágenes sobre personas trans mirando hacia ellas en lugar de mirar con ellas, lo que provoca que la representación trans se construya alrededor de imaginarios cisgénero (Copier y Steinbock, 2018).

\section{Resultados}

Con respecto a la representación de los personajes de Valeria y Cristina Ortíz en la serie de televisión, se incluyen algunas de las variables obtenidas tras la aplicación de la rejilla anterior. En primer lugar, hay que destacar que, en ambos casos, la atracción se presenta únicamente hacia personajes cisgénero, en este sentido, además, tanto en una como en otra ocasión todas las relaciones que se muestran son heterocéntricas. No obstante, en el caso de Valeria, en una de sus relaciones ocasionales, hay una excepción de apenas diez segundos ${ }^{3}$ donde se intuye un encuentro eventual con una mujer. Con respecto a Cristina Ortíz, todas sus relaciones ocasionales y sostenidas son heteronormativas y cisnormativas (Baril y Trevenen, 2014).

En segundo lugar, la violencia es un elemento clave, ya sea en el rol de víctimas o provocadoras. Ese doble papel hace que la violencia se represente en la configuración de las protagonistas y aparezca a distintos niveles, siendo el más representativo el de las microagresiones que las personas trans comúnmente sufren en distintos ámbitos de su vida como el trabajo, su familia o el sistema sanitario, entre otros (Nordmarken, 2014).

La violencia fisica es representada de forma gradual en torno a Cristina Ortíz. Si bien al comienzo $(1 \times 01)$ es obligada a entrar en un vehículo forzada por unas periodistas, más adelante la violencia se vuelve más agresiva con la representación del ataque de un grupo neonazi al colectivo de prostitutas $(1 \mathrm{x} 04)$, al igual que se muestra la violencia de género dentro de la pareja $(1 \mathrm{x} 05,1 \mathrm{x} 06,1 \mathrm{x} 07)$. Junto a ello, son re-

${ }^{3}$ Se puede localizar en el episodio cuarto de la primera temporada (01:32). 
producidos los abusos, violaciones y agresiones que sufre en su estancia en la cárcel (1x07). Sin embargo, la violencia no solo es una cuestión física, sino que también se distingue a nivel psicológico a través de las humillaciones y el rechazo de parte de su familia, en especial de su madre. Microagresiones como dirigirse a las personas trans con los nombres relativos a su género asignado al nacer (Nordmarken, 2014), tal y como hace la madre de Cristina durante toda la serie recordando a Joselito. Asimismo, Cristina mantiene una conversación con su amiga Fani, instantes después de ser víctima de una agresión por parte de su pareja y esta le aconseja volver con su agresor por considerarlo la única opción posible.

Frente a esto, el personaje de Valeria funciona como contrapunto diacrónico de ella, dado que esta no es víctima de agresiones fisicas o psicológicas. Sin embargo, la violencia estructural sistémica es algo que sí está presente en ambas. Desde la escena de presentación de Valeria (que se analizará con más detalle a continuación) hasta su visita a la doctora, donde le insta a la necesidad imperante de una vaginoplastia tras su operación de pecho, afirmando: "cuanto antes hagamos todo mejor». Y es que no se puede olvidar que son narrativas médicas, de discriminación, de violencia, de miedo, de vínculos y afectos; narrativas de frustración por un cuerpo extraño, de luchas e intervenciones indispensables en dicho cuerpo reconfigurado (García, 2018). En este sentido, es reseñable el cuestionario médico previo a la entrada de Cristina en la cárcel de hombres, donde también será el centro de múltiples agresiones, fisicas, sexuales y psicológicas. No obstante, aunque la violencia estructural sistémica está presente en ambos personajes, en Cristina Ortíz se representa de forma más explícita. Esta pierde su trabajo como celadora en un hospital y la justificación de su despido está condicionada por su decisión a transitar. "In our society, it is considered acceptable to be masculine, but to give up that masculinity and present as being feminine is a sign of deviance or some form of mental disorder» ${ }^{4}$ (Reitz, 2017, p. 2). Es a partir de este instante cuando comienza a ejercer de prostituta. Este tipo de construcción junto al hecho de que la totalidad de personajes trans - a excepción de Valeria-, se dediquen a la prostitución, acaba perfilando el trabajo sexual como la única posibilidad laboral para el colectivo.

Esta organización del entorno del personaje trans como violento, se reconfigura y explicita también al convertirse en provocadoras de violencia, dejando en ambos casos a la violencia física como la única que son capaces de ejercer. Sin embargo, en algunas ocasiones la violencia recibida se relaciona de forma cuestionable dado que se presenta como respuesta a la violencia que ellas han provocado, en la misma o en anteriores escenas. «It reinforces negative stereotypes about trans women and makes

\footnotetext{
4 «En nuestra sociedad, está considerado aceptable ser masculino, pero dejar de lado esa masculinidad y presentarse como femenino es un signo de desviación o alguna forma de desorden mental» (Reitz, 2017, p. 2). Traducción propia.
} 
it seem like it is acceptable to treat trans women in the same manner» ${ }^{5}$ (Reitz, 2017, p. 3). De este modo, esta relación causal fortalece el binomio trans/violencia que diseña un imaginario conflictivo para las protagonistas.

La construcción de las relaciones familiares también comparte características comunes. La figura del padre ausente es mutua; en el caso de Valeria por omisión en la trama y en el de Cristina por inacción frente a las decisiones maternas. Para Cristina, la familia es un trauma que solo es superado cuando sustituye a una familia por la otra —en referencia a sus amigas-. Precisamente esta sustitución provocará que la mayor parte de las escenas de grupo en la que participan tanto una como otra sean con mujeres trans, lo que fortalece la idea de gueto o lobby. La dificultad de verlas en grupos diversos fortalece la idea de subgrupo desplazado y periférico ya que son escasas e infrarrepresentadas las escenas de grupo que incluyen a otros personajes cisgénero. Una de ellas es la del encuentro con la familia de Miguel, su pareja, donde se subraya la violencia en las rutinas de las interacciones sociales cuando la suegra considera la identidad trans de la protagonista como algo negativo. "These denigrations are often active manifestations of derogatory stereotypes. Invisible to many deliverers and recipients, they reproduce oppression on the interpersonal level» ${ }^{6}$ (Nordmarken, 2014, p. 129).

La escena de presentación es uno de los momentos más relevantes en la construcción de la protagonista ya que en ella la audiencia debe entender la focalización que tendrá el personaje en la historia. No es casual que muchos manuales de guion (Mckee, 2011; Seger, 2000, Sánchez-Escalonilla, 2016) insistan en su relevancia. En la construcción de personajes trans desde la ficción se hace hincapié en algunos elementos que define Danielle M.Seid bajo el término reveal y que se utilizan en el presente análisis para observar la primera aparición en pantalla de ambos personajes. Asimismo, se aplicarán también los conceptos empleados por Copier y Steinbock (2018) respecto a la composición y las técnicas cinematográficas en la realización visual del encuadre.

En el caso de Valeria se sitúa en su dormitorio, frente al espejo, y se resuelve técnicamente con dos planos medios cortos de un marcado valor simbólico. En el primero de ellos se quita la camiseta y el encuadre decide ocultar el pecho del personaje, cortando la composición a la altura de sus hombros. En cambio, en el segundo

\footnotetext{
5 «Refuerza estereotipos negativos sobre las mujeres trans y hace parecer aceptable el hecho de tratar a las mujeres trans del mismo modo» (Reitz, 2017, p.3). Traducción propia.

6 «Estos actos denigrantes son a menudo manifestaciones activas de los estereotipos despectivos. Estereotipos que son invisibles tanto para las personas a los que se asocian como a aquellas que los perpetúan, reproduciendo así opresión a nivel interpersonal» (Nordmarken, 2014, p.129). Traducción propia.
} 
sí se descubre el torso del personaje, frente al espejo, un objeto fetiche en la representación de personajes trans. El espejo representa 'el otro', alegoría de la disforia de género, presente en el conflicto germinal de la persona trans.

Asimismo, tras el espejo, el resto de los planos impiden reconocerla, lo que define una intencional ambigüedad genérica. Son planos detalles de unas zapatillas moradas pedaleando, planos medios de la espalda mientras monta en bicicleta - donde se puede ver el pelo largo al viento-, y un plano secuencia que empieza con un plano general a contraluz, donde es dificil distinguirla más allá de su silueta, y que concluye con su llegada a la universidad. Si bien, estos primeros cinco planos ayudan a mirarla desde su punto de vista. Es a partir de aquí cuando se presenta al personaje en su contexto. La escena comienza con un plano picado que da origen a la secuencia que recorre la entrada en la universidad de Valeria hasta el aula. En este se puede ver al alumnado que modifica la dirección de su mirada para fijarse en ella. La audiencia es capaz de sentir cómo Valeria se convierte en el centro de todas las miradas. El plano es interrumpido al corte a través del montaje con un plano medio corto de Valeria con la mirada perdida para encadenarse con un plano subjetivo. Aquí se produce un interesante montaje que nos devuelve a Valeria como protagonista para remarcar que es ella quien está mirando.

La posibilidad de ver a través de los ojos Valeria es una diferencia sustancial en cuanto a su representación. Autoras como J. Halberstam lo definen como la mirada transgénero, «opens the door to a nonfetishitic mode of seeing the transgender body - a mode that looks with, rather than at, the transgender body and cultivates the multidimensionality of an indisputably transgender gaze» ${ }^{7}$ (Halberstam, 2005, p.100). Es por lo tanto una representación positiva, ya que frente a ser un elemento feticheobjeto, pasa a ser el sujeto que mira. De este modo, si para Laura Mulvey (1975) la única posibilidad de acceso femenino al cine es de forma voyeurística - frente a la fetichizada - a través de la identificación con los personajes masculinos, esto se traslada a la construcción de personajes trans a través de una mirada cisgénero, algo que refuerza este carácter voyeur en la mirada. Con respecto a Cristina y volviendo a la descomposición visual de Copier y Steinbock (2018), la secuencia comienza con una figura desenfocada, seguida de la inserción de un plano-contraplano muy significativo donde una periodista le dice a su compañero: "coño, Pablo, mira", haciendo referencia al plano completo donde se observa a «Veneno» caminando con el rostro oculto por la iluminación y que refuerza así el concepto voyeurístico. La realización de la secuencia se desarrolla en una consecución de varios planos que recorren el cuerpo de Cristina deteniéndose especialmente en sus pechos. El cambio de for-

\footnotetext{
7 «abre la puerta a un modo de ver el cuerpo trans alejado del fetichismo — un modo de mirar con, en lugar de hacia el cuerpo trans - y cultiva la multidimensionalidad de una mirada trans indisputable» (Halberstam, 2005, p.100).
} 
mato a 4:3 consolida la idea de mirada construida. Igualmente, la periodista le pregunta directamente: "¿tú qué eres, hombre o mujer?», subrayando la idea binaria de la construcción de dos géneros como únicos. La secuencia continúa haciendo hincapié de forma narrativa en la genitalidad explícitamente, así como en los detalles morbosos acerca del trabajo sexual de la protagonista.

Con respecto a las corporalidades, la representación de personajes trans en pantalla fluctúa entre la idea estereotípica de 'estar en el cuerpo equivocado', la 'disforia de género' y el fetiche o espectáculo. (Engdahl, 2014; Keegan, 2013; KochRein et al., 2020; Lovelock, 2017). Es por ello que se considera relevante el analizar la construcción de las protagonistas a través de una escena donde tienen relaciones sexuales con otros personajes.

En el caso de Valeria, hay tres secuencias relevantes donde ella tiene relaciones sexuales con otros personajes $(3 \mathrm{x} 01 / 6 \mathrm{x} 01)$, de las que se seleccionan las pertenecientes al capítulo 3 por su relevancia argumental. En este capítulo, se representa el tránsito de Valeria y se muestra su operación de pecho. Tras esto, la doctora propone una fecha para llevar a cabo la operación de reasignación genital, situación que funciona como detonante de las dudas de la protagonista hacia su cuerpo y sexualidad. Tras el consejo de sus amigas trans de que conozca su cuerpo, Valeria decide quedar con un hombre cisgénero para tener relaciones sexuales. La secuencia está rodada al completo desde un punto de vista alejado del fetichismo, que mira a ambos personajes por igual (personaje trans y personaje cisgénero) (Halberstam, 2005; Steinbock, 2017).

A nivel narrativo se observa cómo se besan en la cama de la habitación. El contacto físico se resuelve con el montaje de primeros planos y se perpetúa la filmación tradicional del cuerpo en pantalla a través de momentos e intensidades en fragmentos (Heath, 1981). En uno de los planos cortos él le toca la entrepierna a Valeria y ella retira su mano, como referencia sutil a la genitalidad y la idea de 'estar en el cuerpo equivocado' que a veces provoca que algunas personas trans se muestren rehacías a tener encuentros y relaciones sexuales, por miedo o vergüenza a la reacción de la otra persona (Cotten, 2014). No obstante, la genitalidad no se muestra de forma explícita en ningún momento.

Después de esta secuencia, es interesante cómo se representa el cambio de Valeria respecto a la confianza y seguridad en su cuerpo y sexualidad, a través de una secuencia donde Valeria tiene relaciones sexuales con otras personas. En la primera secuencia es el hombre el que le abre la puerta a ella y Valeria aparece de espaldas a la cámara totalmente desenfocada, y en este caso es Valeria la que, totalmente enfocada, abre la puerta al hombre — el cuál aparece desenfocado-, subrayando así la importancia del personaje trans en escena tomando un rol activo (Copier y Steinbock, 2018). 
Durante la relación sexual, ahora la atención se pone en Valeria y su placer sexual con una sucesión de planos cortos donde se enmarca al personaje de espaldas a la cámara, con el torso desnudo, y posteriormente se la enmarca de perfil dejando visible los pechos de ella, utilizado comúnmente como signo de feminidad y sexualidad de la mujer, así como objeto fetiche (Young, 2005). La narrativa del capítulo 3 gira en torno al tránsito de Valeria, y su sexualidad.

En este sentido, su tránsito se representa en la serie de forma positiva, sin hacer referencia al hecho de sentirse completa por llevar a cabo la operación de reasignación genital impuesta desde el sistema, sino por llegar a conocerse a sí misma, su cuerpo y su sexualidad, incluso cerrando el capítulo con ella teniendo un orgasmo. Con esto se refuerza la idea esbozada por Galofre y Missé (2015) de una visibilidad positiva que consolide la autoestima y elimine el estigma asociado a las personas trans y su corporalidad.

Respecto a Cristina y su representación durante la primera secuencia, donde ella tiene relaciones sexuales con su pareja (5x01), en el primer plano se enmarca a Angelo de espaldas sentado en la cama. Hay un travelling hacia él conforme va girándose y dirigiendo la mirada hacia la puerta — donde aparece Cristina posteriormente- generando así la sensación en la audiencia de querer verlo todo y conocer más sobre la información oculta e insinuada por la mirada del personaje cis (Heath, 1981; Seid, 2014). Esta idea de revelación de información se ve incrementada por el siguiente plano medio donde aparece Cristina Ortíz entrando en la habitación a oscuras hasta que se coloca en un haz de luz que la ilumina dejando ver su torso desnudo y su ropa interior - sin mostrar su genitalidad-. Así, se presenta el cuerpo del personaje trans a través de la narrativa del espectáculo. Concepto que Koch-Rein et al. (2020) destacan como la mirada cinematográfica que exhibe el cuerpo trans, reduciéndolo solo a eso. Mirada que se refuerza con el contraplano siguiente donde se descubre de nuevo a Angelo observándola y diciendo: "Sei bellisima». A continuación, se vuelve a enmarcar a 'Veneno' en el foco de luz, esta vez tocándose los pechos, siendo este uno de los principales aspectos para situar a la mujer como objeto en pantalla (Young, 2005). En este mismo encuadre, Angelo se baja los pantalones y ella dirige su mirada hacia sus genitales, siendo esta la única referencia implícita a la morfología sexual, en este caso sobre el personaje cis.

Toda la acción se presenta en una sucesión de tomas muy cortas con una proyección de ritmo rápido y una angulación oblicua. Técnicas que se alejan de la tradición filmica que construye el espacio a través de un proceso de 'sutura' gracias al montaje clásico con recursos como el del campo-contracampo (Halberstam, 2005; Heath, 1981).

En este sentido, aunque se perpetúa la técnica de fragmentación de cuerpos (Heath, 1981) y la lógica filmica de corporalidad trans acuñada por Susan Stryker 
(2013), lo más relevante es cómo esos fragmentos son tratados en relación con la coherencia y construcción del personaje trans (Copier y Steinbock, 2018), que en este caso se torna como protagonista y encarna un rol activo durante el acto sexual, sin filmación sobre su genitalidad y poniendo el foco tanto en sus pechos como en su rostro. Rostro que además cierra la secuencia, con un primer plano donde ella tiene un orgasmo.

\section{Conclusiones}

En síntesis y tras lo observado en los resultados, se localizan algunas tendencias en la construcción de personajes trans que ayudan a confirmar los objetivos planteados al inicio de este trabajo. La representación de las protagonistas 'Veneno' y Valeria se sostiene en varios elementos identitarios que configuran la disposición que tiene la audiencia al acercarse a ellas. Entre estos, se halla una tendencia común a reproducir una orientación sexual de las mujeres trans hacia hombres, mostrando así una sexualidad canónica basada en un sistema heteronormativo, fortalecido además por el hecho de que los hombres tengan una identidad cisgénero. Esta representación de relaciones exclusivamente con hombres cisgénero se ve paradójicamente enfrentada con la construcción de las relaciones amistosas y familiares las cuales se establecen con otras mujeres trans, fomentando así la construcción de gueto asociada a las protagonistas. Respecto a los tropos negativos, en primer lugar, se construye a los personajes trans en un contexto de violencia (estructura/sistémica, física o psicológica) donde ellos mismos como actantes se encuentran constantemente en una situación de crisis. Otra de las tendencias negativas que se perpetúan reside en la manifestación de las posibilidades sociolaborales vinculadas inevitablemente con el trabajo sexual, así como la representación del cuerpo de 'Veneno' en torno a una narrativa del espectáculo, como objeto fetiche. En concordancia con ello, se construye un tipo de focalización externa a la hora de presentar a ambas protagonistas, vinculada al concepto voyeurístico, es decir mirar desde fuera en lugar de mirar con ellas. Algo que se refuerza por la metadiscursividad televisiva de la serie y la constante puesta en abismo que incluye el dispositivo, al igual que por el uso mayoritario de técnicas tradicionales como el uso del plano-contraplano. No obstante, esta focalización externa se ve sustituida por una 'mirada transgénero' en algunas ocasiones a través del personaje de Valeria, la cual además se desvincula del tropo negativo del trabajo sexual. Por otro lado, desde la construcción de Valeria se observa una tendencia alejada de la tradición estereotípica en torno a la identidad y corporalidad, asumiendo un rol activo a la hora de tomar sus propias decisiones respecto a su sexualidad y transición.

En resumen, a través del presente estudio de caso y las escenas claves seleccionadas, se observa un fomento de la visibilidad del colectivo trans en la serie Veneno (2020). No obstante, aunque a través del análisis del personaje de Valeria se advierten ciertos aspectos de representación positiva, estos se ven justificados por la dife- 
rencia temporal y su papel como contraposición diacrónica de 'Veneno', la cual se construye con una gramática cinematográfica tradicional y se presenta desde una perspectiva cisgénero que en lugar de mirar con ella la sitúa ante la audiencia para que sea observada. Respecto a la corporalidad además, se perpetúa la tradición normativa que sitúa a las personas en categorías estancas, como si no hubiera posibilidad de estar de forma completa en una identidad que transgreda lo establecido, como si las lógicas binarias impulsaran a los cuerpos a situarse en los extremos. El análisis más exhaustivo de las diferentes corporalidades construidas, abre un espacio de debate e investigación amplio donde se podría aplicar una perspectiva más materialista y afectiva alejada del nivel más narrativo y basado en estereotipos, que ponga el foco en la relación entre cuerpos e identidades en pantalla, y la posibilidad de construir nuevas narrativas, tal y como señalaba Sedgwick (1997), desde una lectura reparativa que reseñe los aspectos positivos y transgresores del audiovisual.

\section{Contribuciones específicas y orden de autoría en el artículo}

En el presente artículo ambo/as autore/as han contribuido de forma equitativa. El criterio para el orden de autor y autora se ha basado en el orden alfabético de los apellidos, así como las responsabilidades éticas contraídas.

\section{Referencias}

Adair, C., Awkward-Rich, C., y Marvin, A. (2020). Before Trans Studies. TSQ: Transgender Studies Quartely, 7(3), 306-320. https://doi.org/10.1215/23289252-8552922

Baril, A. y Trevenen K. (2014). Exploring ableism and cisnormativity in the conceptualization of identity and sexuality 'disorders'. Annual Review of Critical Psychology, 11(1), 389-416.

Bernini, L. (2018). Las teorías queer. Una introducción. Barcelona: Egales.

Butler, J. (2006). Deshacer el género. Paidós.

Branigan, E. (1984). Point of View in the Cinema: A Theory of Narration and Subjectivity in Classical Film. Mouton Publishers.

Carter, J. (2014). Transition. TSQ: Transgender Studies Quarterly, 1(1-2), 235-237. https:// doi.org/10.1215/23289252-2400145

Casetti, F., \& Di Chio, F. (2007). Cómo analizar un film. Paidós Comunicación 172 Cine.

Cobo-Durán, S. (2011). Uso de roles en la construcción de personajes: desde la Nueva Masculinidad a los estereotipos de género. En Pérez-Gómez, M.A. (Ed.), Previously on: estudios interdisciplinarios sobre la ficción televisiva en la Tercera Edad de Oro de la Televisión. (pp.585-597). Biblioteca de la Facultad de Comunicación de la Universidad de Sevilla. 
Collazos, W. P. (2009). El estudio de caso como recurso metodológico apropiado a la investigación en ciencias sociales. Educación y desarrollo social, 3(2), 180-195. https://doi. org/10.18359/reds.887

Copier, L. y Steinbock, E. (2018). On not really being there: trans` presence/absence in $D a$ llas Buyers Club, Feminist Media Studies, 18(5), 923-941. https://doi.org/10.1080/1468 0777.2017.1393833

Córdoba, D., Sáez, J. y Vidarte, P. (2005). Teoría queer. Políticas bolleras, maricas, trans, mestizas. Egales.

Cotten, T. T. (2014). Surgery. TSQ: Transgender Studies Quarterly, 1(1-2), 205-207. https:// doi.org/10.1215/23289252-2400028

Crenshaw, K. (1991). Mapping the Margins: Intersectionality, Identity Politics, and Violence against Women of Color. Stanford Law Review, 43(6), 1241-1299. https://doi. org/10.2307/1229039

Cuevas, E. (2001) Focalización en los relatos audiovisuales. Trípodos, 11, 123-136.

Dubrofsky, R. (2013). Jewishness, whiteness, and blackness on glee: Singing to the tune of postracism. Communication, Culture \& Critique, 6, 82-102. https://doi.org/10.1111/ cccr. 12002

Engdahl, U. (2014). Wrong body. TSQ: Transgender Studies Quarterly, 1(1-2), 267-269. https://doi.org/10.1215/23289252-2400226

Enke, A.F. (2012). Introduction Transfeminist Perspectives. En A.F. Enke (Ed.), Transfeminist Perspectives. In and beyond Transgender and Gender Studies. (pp.1-15). Temple University Press.

Fellner, A. (2017) Trans Television Culture. Queer Politics, Gender Fluidity, and Quality TV. Oceánide, 9.

Galofre, P. y Missé, M. (2015). Políticas trans. Una antología de textos desde los estudios trans norteamericanos. Barcelona-Madrid: Egales.

García, A. (2018). Tacones, siliconas, hormonas: Etnografía, teoría feminista y experiencia trans. Bogotá: Siglo del hombre editores.

Garner, T. (2014). Becoming. TSQ: Transgender Studies Quarterly, 1(1-2), 30-32. https://doi. org/10.1215/23289252-2399515

Genette, G. (1998). Nuevo discurso del relato. Cátedra.

Gillig, T.K., Rosenthal, E.L., Murphy, S.T. y Langrall, K. More than a Media Moment: The Influence of Televised Storylines on Viewers' Attitudes toward Transgender People and Policies. Sex Roles, 78, 515-527. https://doi.org/10.1007/s11199-0170816-1

Green, E.R. (2006). Debating Trans Inclusion in the Feminist Movement. Journal of Lesbian Studies, 10(1-2), 231-248. http://dx.doi.org/10.1300/J155v10n01_12

Greimas, A. (1971). Semántica estructural. Gredos.

Halberstam, J. (2005). In a queer time and place. Transgender bodies, subcultural lives. Nueva York-Londres: New York University Press. 
Halberstam, J. (2016). Chapter 7. Transgender in a Global Frame. En Horlacher, S. (coord.), Transgender and Intersex: Theoretical, Practical, and Artistic Perspectives (pp. 165-187). Nueva York: Palgrave Macmillan US.

Heath, S. (1981). Questions of Cinema. Bloomington: Indiana University Press, 1981.

hooks, b. (1984). Feminist Theory: From Margin to Center. Boston: South End Press Classics.

Horak, L. (2018). Trans Studies. Feminist Media Histories, 4(2), 201-206. https://doi. org/10.1525/fmh.2018.4.2.201

Keegan, C. M. (2013). Moving bodies: Sympathetic migrations in transgender narrativity. Genders, 57.

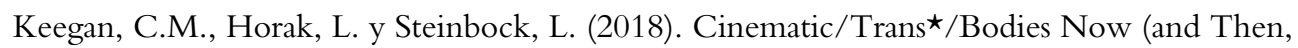
and to Come). Somatechnics, 8(1), 1-13. https://doi.org/10.3366/soma.2018.0233

Koch-Rein, A., Haschemi, E., y Verlinden, J. J. (2020). Representing trans: Visibility and its discontents. European Journal of English Studies, 24(1), 1-12. https://doi.org/10.1080/1 3825577.2020 .1730040

Kociemba, D. (2010). "This isn't something I can fake»: Reactions to Glee's representations of disability. Transformative Works and Cultures, 5. https://doi.org/10.3983/ twc. 2010.0225

López, M.A. (2017). Heternormatividad. En R.L. Platero, M. Rosón y E. Ortega (Eds.), Barbarismos queer y otras esdrújulas (pp.228-238). Bellaterra.

Lovelock, M. (2017). Call me Caitlyn: Making and making over the «authentic» transgender body in Anglo-American popular culture. Journal of Gender Studies, 26(6), 675-687. https://doi.org/10.1080/09589236.2016.1155978

Madrigal, L. (2021). El lenguaje queer en versión original y traducida. Una comparación entre Veneno y RuPaul's Drag Race. [Trabajo Fin de Grado no publicado]. Universidad de Alicante.

Mckee, R. (2011). El guión. Story. Madrid: Alba editorial.

Mulvey, L. (1975). Visual Pleasure and Narrative Cinema. Screen, 16(3), 6-18. https://doi. org/10.1093/screen/16.3.6

Navarro, A. (2021). La ficción audiovisual como representación de la comunidad trans: análisis de la cobertura mediática nacional de la serie 'Veneno'. [Trabajo Fin de Grado no publicado]. Universidad de la Laguna.

Nordmarken, S. (2014). Microaggressions. TSQ: Transgender Studies Quartely, 1(1-2), 129134. https://doi.org/10.1215/23289252-2399812

Platero, R.L. (2014). Trans ${ }^{\star}$ exualidades. Acompañamiento, factores de salud y recursos educativos. Bellaterra.

Propp, V. (1998). Morfología del cuento. Ediciones Akal.

Reitz, N. (2017). The Representation of Trans Women in Film and Television. Cinesthesia, $7(1)$.

Rodemeyer, L.M. (2018). Feminist and Transgender Tensions: An Inquiry into History, Methodological Paradigms, and Embodiment. En C.Fischer y L.Dolezal (coords.) New Feminist Perspectives on Embodiment (pp.103-123). Cham: Palgrave Macmillan US. 
Sánchez-Escalonilla, A. (2016). Del guion a la pantalla. Lenguaje visual para guionistas y directores de cine. Ariel.

Sandercock, T. (2015). Transing the small screen: loving and hating transgender youth in Glee and Degrassi. Journal of Gender Studies, 24(4), 436-452. https://doi.org/10.1080/ 09589236.2015.1021307

Sears, C. (2018). All that glitters: Trans-ing California's gold rush migrations. GLQ: A Journal of Lesbian and Gay Studies, 14, 383-402. https://doi.org/10.1215/10642684-2007-038

Sedgwick, E.K. (1997). Paranoid Reading and Reparative Reading; or You're So Paranoid, You Probably Think This Introduction is About You. En Sedgwick, E.K. (Ed.), Novel Gazing: Queer Readings in Fiction (pp.123-151). Duke University Press.

Seger, L. (2002). Cómo crear personajes inolvidables: guía práctica para el desarrollo de personajes en cine, televisión, publicidad, novelas y narraciones cortas. Madrid: Paidós Ibérica.

Seid, D. M. (2014). Reveal. TSQ: Transgender Studies Quartely, 1(1-2), 176-177.

Steinbock, E. (2016). Trans. En R.Hoogland, I. van der Tuin y N. Fleetwood (coords.), Gender: Sources, Perspectives, Methodologies (pp.377-392). Farmington: Macmillan Reference USA.

Steinbock, E. (2019). Towards Trans Cinema. En K. L. Hole, D. Jelaca, E. A. Kaplan, y P. Petro (coords.), The Routledge Companion to Cinema and Gender (pp. 395-406). Londres-Nueva York: Routledge.

Stone, S. (1992). The Empire Strickes Back: A Posttransexual Manifesto. Camera Obscura. Feminism, culture, and media studies, 10(2), 150-176. https://doi.org/10.1215/0270534610-2_29-150

Stryker, S. (2006). (De)Subjugated Knowledges. An Introduction to Transgender Studies. En S. Stryker y S. Whittle (coords.), The Transgender Studies Reader (pp. 1-18). Nueva York: Routledge.

Stryker, S. (2013). Christine in the Cutting Room: Cinema, Surgery, and Celebrity in the Career of Christine Jorgensen. Department of Media, Music, Communication and Cultural Studies Public Lecture Series. Disponible en https://www.youtube.com/ watch? $\mathrm{v}=\mathrm{XlqJ} 8 \mathrm{~B} 9 \mathrm{dKCs} \& \mathrm{t}=951 \mathrm{~s}$

Stryker, S. (2017). Historia de lo trans - Las raíces de la revolución de hoy. Madrid: Continta Me Tienes.

Stryker, S. y Bettcher, T.M. (2016). Introduction. Trans/Feminisms. TSQ: Transgender Studies Quarterly, 3(1-2), 5-14. https://doi.org/10.1215/23289252-3334127

Torras, M. (2017) Embodiment (embodimén). En R.L.Platero, M.Rosón y E.Ortega (Eds.), Barbarismos queer y otras esdrújulas (pp.161-167). Bellaterra. s

Young, I.M. (2005). Breasted experience: The look and the feeling. En I.M. Young (coord.). On female body experience: Throwing like a girl and other essays (pp.75-96). Nueva York: Oxford University Press.

Zurián, F. A. (2018). Representaciones LGTBIQ en la televisión de ficción española, de la Transición a Zapatero. En Ingenshay, D. (Ed.), Eventos del Deseo. (pp. 243-261). Editorial Iberoamericana-Vervuert. 